\title{
Editorial
}

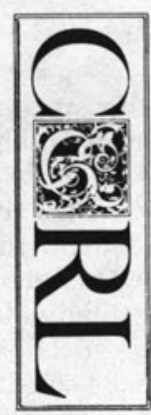

\section{Improving VDT Work}

Do your eyes feel blurred, watery, or tired during or after use of the VDT (video display terminal)? Do your shoulders and neck feel strained? The answer to both of these questions is yes for many if not most VDT users. My answer is yes and I am not a heavy user. The answer to my problem is at least partially within my control: I need reading glasses. My VDT sits on top of my $291 / 2$-inch-high desk, a poor height according to published standards. The proper height for a nonadjustable VDT table is about 25 to 26 inches. My table is higher. My table is also not adjustable, a feature that would become necessary if I used the VDT for long periods or if other operators also used the same VDT. (Chair height in relationship to desk height is a key variable).

In the November 20,1985, issue of the Chronicle of Higher Education it was reported that campus clerical workers are increasingly concerned about health factors related to the automatic office equipment they use. Minolta polled 800 members of Professional Secretaries International for their opinions about VDT use. Members reported many problems: eyestrain (52 percent), back or neck strain (46 percent), headaches ( 30 percent), and other types of stress ( 23 percent).

In a survey by Verbatim Corporation, covering 1,263 office workers in large, mediumsized and small firms nationwide, heavy terminal users wanted better lighting ( 79 percent) and more rest breaks ( 78 percent). Eye- and back strain were cited as the most important problems.

Library workers share many of the same problems. Russell Gardiner is worried about the staff that he manages in the bibliographic services department at Berkeley. He foresees the potential for eight-hour VDT shifts. Already he encourages staff rotation opportunities. Members of his staff work at the catalog information desk and at the general reference desk.

Coincidentally, one of Russell's supervising library assistants, Mercedes Untawale, coauthored an article with me on "Work Enrichment in Academic Libraries" that appeared in the January 1983 issue of the Journal of Academic Librarianship. In this article we pointed out the need to examine the design of work when faced with the implementation of an automated system. Too often good work-design principles are ignored and psychological stress is followed by physical stress. This phenomenon has been called technostress: a result of the inability to cope in a healthy manner with the various manifestations of a technology.

The design of work is a complicated process. The process of improving VDT work is straightforward in comparison. Ambient lighting can be measured. The height of a table can be measured. The components of an effective work-station environment can be blueprinted. In a manual, Improving VDT Work: Causes and Control of Health Concerns in VDT Use, prepared by the Wisconsin Department of Administration (distributed by the Report Store in Lawrence, Kansas), Steven Sauter "challenges the notion that VDT work must be necessarily associated with discomfort."

In the manual, many practical, low-cost measures are recommended. There are six spe- 
cific suggestions for alleviating problems related to the back, the neck and shoulders, the arms and hands, the legs, the characteristics of the equipment, and the eyes.

- Identify and focus upon the jobs and work stations most in need of attention.

- Insure that new equipment, chairs, tables, and displays have a wide variety of easy adjustments.

- Do not tolerate a display with an unsteady image or legibility problems.

- Eliminate reflections and glare with proper room lighting; use screen filters to control reflections as needed.

- Be sensitive to psychological or personnel traits that may be contributing to job stress.

- Train operators in the proper use of equipment and personal habits related to their comfort at work.

Libraries and their respective campuses should develop programs to help the employee to learn how to relieve the various types of physical strain that are caused by VDT work.

It is my personal belief that every library should conduct an audit of every VDT work station in order to compare current conditions against current standards. This would include the video display, lighting control, ergonomics of the work station, and other factors that may affect the health of the employee-user work stations should not be ignored. A list should be prepared of conditions that do not meet standards. A short-term plan should then be prepared to bring listed items into conformity with standards.

If libraries can afford to buy expensive automated systems, they can certainly afford to provide optimum working conditions.

CHARLES MARTELL

\section{IN FORTHCOMING ISSUES OF COLLEGE \& RESEARCH LIBRARIES}

Papers presented at a seminar sponsored by the Research Libraries Group, Conoco's Purple

Sage Ranch in Bandera, Texas, November 1985

Open Systems for Open Minds: Building the Library without Walls

by John R. Sack

Libraries as Life-Systems: Information, Entropy and Coevolution on Campus

by Timothy C. Weiskel

Meeting Scholarly Information Needs in an Automated Environment: A Humanist's

Perspective

by David Crawford

Quiescence, Query, Quandary, Quietus: Public Services in the Library of the Future

by Henry Snyder

Evaluation of the Public Service Functions of Serial File Systems

by Elizabeth Hanson and Judith Serebnick

Library Skills, Critical Thinking, and the Teacher Training Curriculum

by Nancyanne O'Hanlon

Foreign Students, Libraries, and Culture

by Mary Alice Ball and Molly Mahoney

Cataloging U.S. Depository Materials: A Reevaluation

by Alice Harrison Bahr

Community College Learning Resources Centers at the Crossroads: Illinois, a Case Study

by Eillen Dubin and Linda Bigelow 